\title{
Aspectos que influyen en las estrategias de marketing de distribución y publicidad en Huancayo, Perú
}

\section{Aspects that influence in the marketing strategies of distribution and advertising in Huancayo, Perú}

José María Matas Castañeda

Universidad Continental

\section{INTRODUCCIÓN}

El propósito de este artículo es analizar la situación actual de las principales estrategias de marketing en las variables de distribución y comunicación de Huancayo, capital del departamento de Junín, una de las más importantes zonas de los Andes Centrales del Perú.

Esta región tiene como ciudad próxima de importancia a la ciudad de Lima, la capital peruana, que se encuentra a unas ocho horas con los medios de transporte actuales, después de atravesar los Andes y llegar hasta la costa. La mayoría de carreteras en los Andes y hasta la selva han sido sin asfaltar y complicadas aun los últimos años, por lo que el desplazamiento entre ciudades lo hacían pocas personas. Ello explica que tenga formas de distribución y comunicación propias.

La llegada del primer centro comercial y la utilización de medios digitales están transformando las formas tradicionales de distribución y comunicación.

El trabajo también analiza cómo se manifiesta la variable de comunicación en el marketing mix de las empresas huancaínas y cómo han influido la llegada de los medios digitales.

1 Lic. en Ciencias Empresariales, máster en Dirección de Empresas por ESADE Business School, Barcelona, España; docente e investigador de la Universidad Continental. 
mitad va a Lima y el resto se queda en la región.

\section{Los mercados}

El mercado Mayorista de Huancayo, junto con el mercado Modelo, que es el mercado central detallista o minorista, conforman el mayor mercado andino del Perú. Ni siquiera en Cusco, ciudad andina de un tamaño similar a Huancayo, hay un mercado tan grande y surtido.

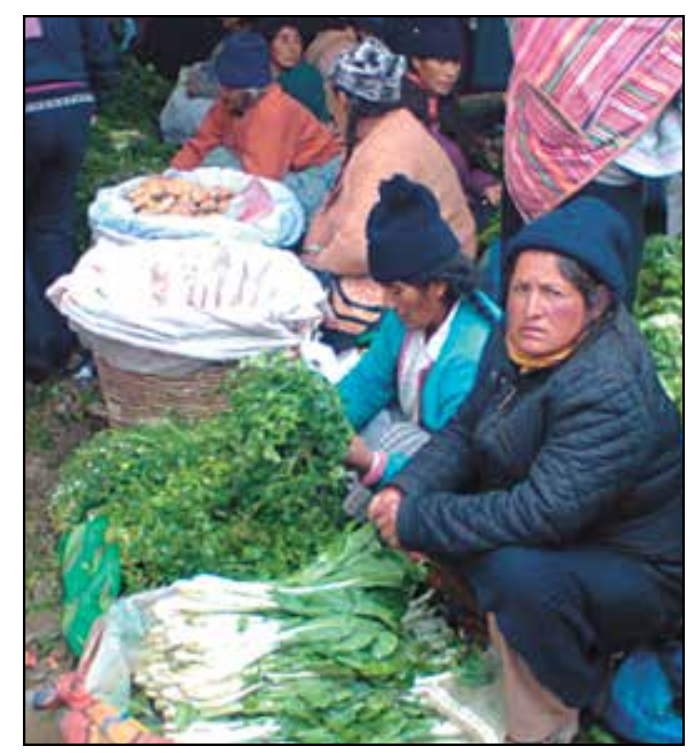

Su ubicación es un lugar central de la ciudad, cuyo eje es la vía del ferrocarril. Además de los propios mercados, hay centenares de puestos informales en los alrededores y por las calles creando un ambiente andino multicolor único donde es ofertada la gran variedad de productos de los Andes y de la Selva Central.

\section{Real Plaza}

Abierto en diciembre de 2008, es el único centro comercial de Huancayo en la actualidad. Incluye un supermercado, tiendas por departamento, sala multicines, restaurantes, patio de comidas, etc. Está muy bien situado en la zona central de la ciudad en el cruce de las calles Giráldez y Ferrocarril, sector muy comercial y junto al Mercado Mayorista.

La novedad del tipo de establecimiento en toda la región ha provocado un traslado de una parte importante de los consumidores locales y de la región atraídos por los tipos de establecimientos y los productos extranjeros a los que no están acostumbrados.

El establecimiento ha influido negativamente en los comercios del centro urbano tradicionales de mayor prestigio de Huancayo, impacto que se manifiesta en la disminución de las ventas en los negocios, en especial en las galerías comerciales locales y en las tiendas de mayor nivel.

También ha afectado al Mercado Mayorista, que se encuentra al lado, y a los mejores puestos y tiendas de la zona del Mercado Modelo. Además, ha generado una mayor centralización de la ciudad y un mayor atasco circulatorio de vehículos.

\section{Hipermercados y supermercados}

En la actualidad hay tres hipermercados $y$ dos supermercados en toda la ciudad.

\section{Las bodegas}

Es el comercio tradicional que existe en todas las calles de Huancayo. Son tiendas que abastecen de todo tipo de productos, principalmente de alimentación. Suelen aceptar el pago aplazado.

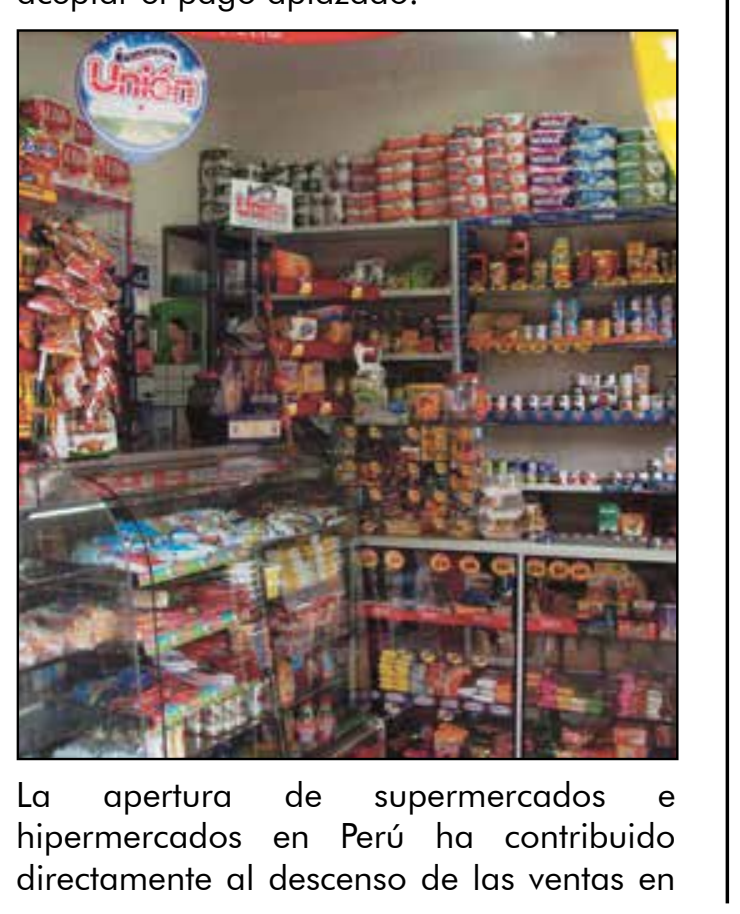




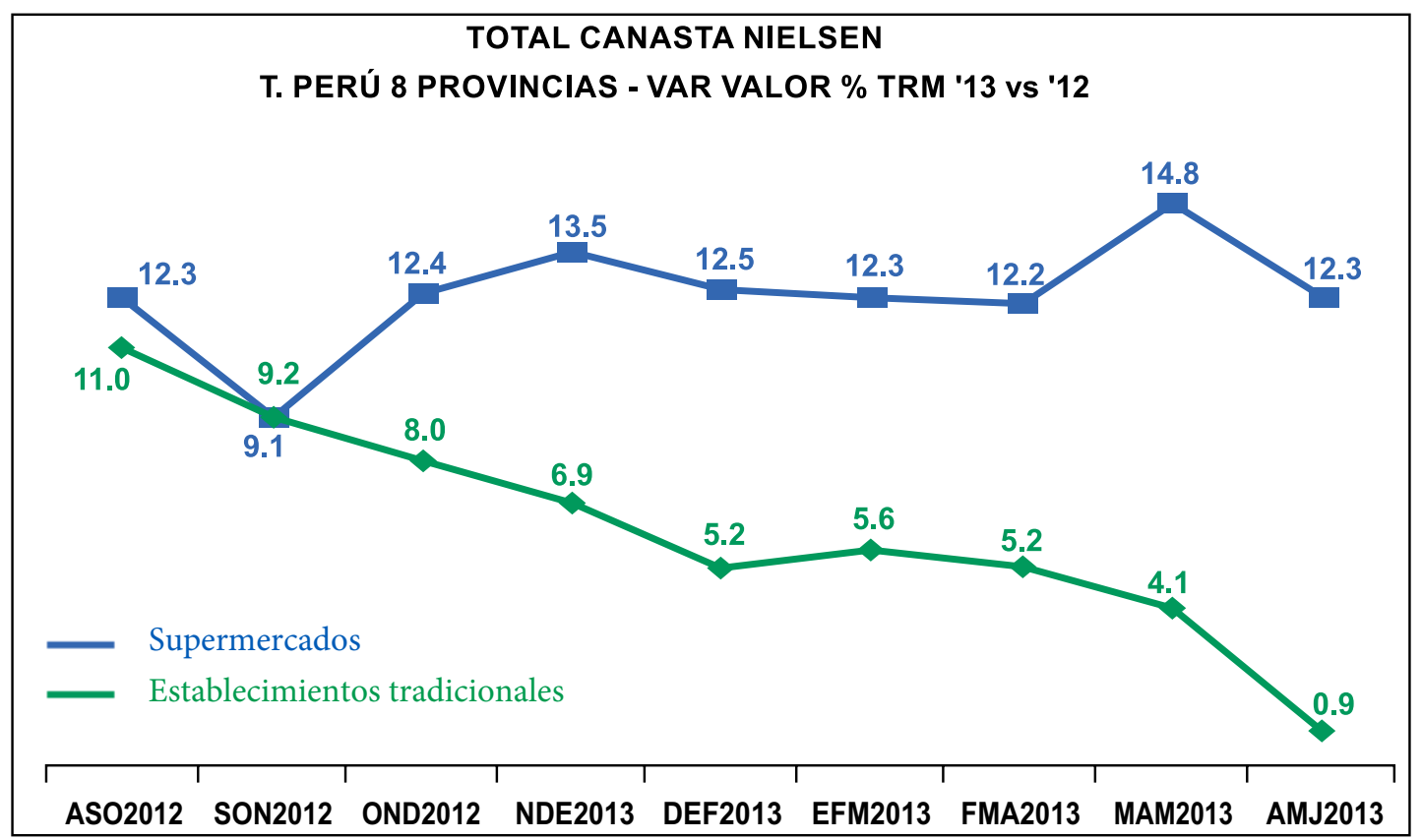

Figura $\mathrm{N}^{\circ}$ 1: Comparación de la variación de las ventas en valor en Perú Fuente: Nielsen

las bodegas y en general en todo el sector tradicional del país (figura $\mathrm{N}^{\circ} 1$ ).

\section{La comercialización gremial}

Muchos de los comercios especializados se juntan en un lugar para ofertar el mismo producto o servicio. Compiten entre ellos y el usuario sabe dónde encontrarlos y así negociar entre los ofertantes.

\section{Algunos ejemplos:}

- Muebles: En la zona ubicada entre las calles Cajamarca y Huancavelica.

- Cortinas: Entre las calles Cajamarca y Arequipa.

- Pinturas: Entre las calles Cajamarca y Ferrocarril.

- Ferreterías: En la calle Tarapacá.

- Llaves: Entre las calles Santa Isabel y Sebastián Lorente.

- Abogados: Entre las calles Nemesio Ráez y Parra del Riego.
- Fotocopias e impresiones: Entre las calles Real y Cusco.

- Ecografías: En la calle Puno, entre Moquegua y Huancavelica.

\section{Los medios empleados}

\section{1) Medios tradicionales:}

Televisión por cable:

La televisión por cable ha cambiado la forma de ver televisión. Considerando a Huancayo con una situación similar a la del interior urbano, habría un 43,5 \% de hogares con televisión por cable, caso que transforma la situación de la audiencia televisiva. A partir de esta nueva situación la audiencia de las televisoras locales viene disminuyendo, ya que quien tiene televisión por cable deja de ver la televisión local.

Los canales de televisión más vistos:

América Televisión, ATV y Panamericana Televisión son los canales nacionales más vistos en Huancayo. El resto de canales son $100 \%$ locales. 
Tabla $N^{\circ} 1$ : Uso de Internet en Perú por sexo y edad (2011-2012)

\begin{tabular}{|c|c|c|c|}
\hline \multicolumn{4}{|c|}{$\begin{array}{c}\text { Perú: Población de } 6 \text { y más años de edad que hace uso de Internet según sexo y grupos de edad } \\
\text { Trimestre Octubre - Noviembre - Diciembre: } 2011-2012 \\
\text { (Porcentaje del total de población de } 6 \text { y más años de edad de cada sexo y grupo de edad) }\end{array}$} \\
\hline Sexo/Grupos de edad & Oct. - Nov. - Dic. 2011 & Oct. - Nov. - Dic. 2012 P/ & $\begin{array}{c}\text { Variación absoluta } \\
\text { (en puntos porcentuales) }\end{array}$ \\
\hline Total & 37,5 & 39,4 & 1,9 \\
\hline Hombre & 41,3 & 42,8 & 1,5 \\
\hline Mujer & 33,6 & 36,0 & 2,4 \\
\hline \multicolumn{4}{|l|}{ Grupos de edad } \\
\hline De 6 a 11 & 29,6 & 30,9 & 1,3 \\
\hline De 12 a 18 & 63,2 & 64,2 & 1,0 \\
\hline De 19 a 24 & 62,8 & 67,2 & 4,4 \\
\hline De 25 a 40 & 37,8 & 41,3 & 3,5 \\
\hline De 41 a 59 & 21,7 & 23,2 & 1,5 \\
\hline de 60 y más & 7,5 & 8,6 & 1,1 \\
\hline
\end{tabular}

P/Preliminar

Fuente: Instituto Nacional de Estadística e Informática - INEI

Tabla $N^{\circ}$ 2: Uso de Internet por nivel educativo (2011-2012)

Perú: Población de 6 y más años de edad que hace uso de Internet según nivel educativo alcanzado Trimestre Octubre - Noviembre - Diciembre: 2011-2012

(Porcentaje del total de población de 6 y más años de edad de cada nivel educativo)

\begin{tabular}{lccc}
\hline Nivel educativo & Oct. - Nov. - Dic. 2011 & Oct. - Nov. - Dic. 2012 P/ & $\begin{array}{c}\text { Variación absoluta } \\
\text { (en puntos porcentuales) }\end{array}$ \\
\hline Total & 37,5 & 39,4 & 1,9 \\
Primaria 1/ & 15,7 & 16,7 & 1,0 \\
Secundaria & 40,3 & 41,5 & 1,2 \\
Superior no universitaria & 58,5 & 62,4 & 3,9 \\
Superior universitaria & 81,2 & 85,2 & 4,0 \\
\hline
\end{tabular}

1/Incluye sin nivel e inicial

$\mathrm{P} /$ Preliminar

Fuente: Instituto Nacional de Estadística e Informática - INEI

\section{Las principales emisoras radiales:}

Radio Huancayo, emisora local, es líder de la audiencia radial, combina noticias locales con música regional. RPP es la emisora radial de mayor alcance nacional con sus informativos de 24 horas diarias. $15-50$ y Nueva $Q$ son las emisoras locales que siguen en preferencia de audiencia en Huancayo.

\section{Prensa}

El diario Correo domina la prensa escrita con 84000 lectores diarios. Su contenido en las primeras páginas es de tipo policial (similar al desaparecido "El Caso" de España) pasando luego a noticias locales.

\section{Revistas locales}

Después de la revista Quince, no hay otras que resaltar.

\section{Vallas}

Situadas en lugares estratégicos.

Afiches, carteles 
Se usan con frecuencia.

Ventas personales

Es el método tradicional de comunicación y el más utilizado.

\section{2) Medios digitales:}

\section{Uso de medios digitales}

El uso de Internet en Perú alcanza en la actualidad (según los estudios del INEI del 2012) al 39,4 \% de la población, cuyo crecimiento en su uso es mayor en la población joven, de 19 a 24 años, que llega al $67,2 \%$ seguido por la población de 6 a 11 años que asciende al 30,9\%, una cifra próxima a la media nacional (tabla $\mathrm{N}^{\circ} 1$ ).

El uso según el nivel de educación indica que los que más utilizan el servicio son los estudiantes de educación superior universitaria $(85,2 \%)$ y no universitaria $(62,4 \%)$, lo que quiere decir que a mayor la formación, mayor uso de los medios digitales (tabla $\mathrm{N}^{\circ} 2$ ).

El uso de las redes sociales en Perú es masivo por parte de los usuarios, hay un fuerte crecimiento en el 2012 respecto al 2011 y el uso en el interior urbano del país está a un nivel próximo al de Lima (tabla

Tabla $N^{\circ}$ 3: Usuarios de redes sociales en Lima y el interior (2011-2012)

\begin{tabular}{lccc}
\hline Perú urbano & Total & Lima & Interior \\
\hline $\begin{array}{l}\text { Usuarios de redes } \\
\text { sociales }(2011))^{* *}\end{array}$ & $60 \%$ & $64 \%$ & $56 \%$ \\
$\begin{array}{l}\text { Usuarios de redes } \\
\text { sociales (2012)** }\end{array}$ & $75 \%$ & $79 \%$ & $71 \%$ \\
\hline
\end{tabular}

$\left.N^{\circ} 3\right)$.

La principal red social en Perú es Facebook $y$ es utilizada por el $97 \%$ de usuarios, seguida por Linkedin (32\%) y Twitter (22\%). Esta red social se utiliza en Huancayo principalmente para las relaciones sociales, no para la comercialización de productos. Aun cuando de comercializar se trata, las empresas prefieren tener una página en Facebook antes que desarrollar una página o sitio web.

No se observan anuncios "pagados" de empresas huancaínas en Facebook, tampoco en Google, el buscador mayoritario.

\section{Uso publicitario}

El uso masivo de Facebook como herramienta de red social hace que las empresas prefieran tener una página de Facebook antes que una página web. En el caso de tener las dos, suelen ser mucho más activos en Facebook que en la página web. La venta personal, medio de comunicación más usado, se complementa con la comunicación directa en el muro del Facebook y la utilización de grupos en dicha red social.

\section{CONCLUSIONES}

Huancayo, principal zona de los Andes Centrales del Perú, está viviendo una transformación en sus formas de comercialización tradicionales. Se crean nuevos conceptos de empresas locales. La llegada del primer centro comercial moderno, Real Plaza, en pleno centro de la ciudad, está transformando el mapa comercial local.

La composición de la distribución en la ciudad de Huancayo viene motivada por los siguientes factores:

- Una economía que ha estado basada en la subsistencia durante siglos, ya que el problema para alimentarse en la zona andina por la irregularidad del terreno y la dificultad de trasladarse de un lugar a otro, ha obligado a sus habitantes a trabajar muy duro para recoger alimentos.

- La ciudad de Huancayo está en el mayor valle de los Andes Centrales, cuya zona de ocupación abarca unos $60 \mathrm{~km}$ de largo y unos 15 de ancho y se trata de un valle muy fértil. Por eso los españoles, 
cuando llegaron a Perú, pusieron la primera capital del país en este valle, en la ciudad de Jauja. Además está en un lugar central de los Andes peruanos y es la ciudad andina más próxima a Lima, la capital actual. Esto hace que la ciudad de Huancayo tenga una cultura distinta a la del resto de los Andes, puesto que tiene la posibilidad de contar con amplios cultivos y concentrar productos para comercializar.

- La combinación de los factores anteriores hace que Huancayo, por un lado, tenga el mayor Mercado Central de los Andes, si consideramos el Mercado Mayorista, el Mercado Modelo y todas las calles que le rodean, y a la vez existe un mercado de bodegas tradicionales muy disperso. El Mercado Central se entiende como centro de distribución andino, y las bodegas, como la sustitución del autoconsumo andino, porque se trata de pequeños comercios que permiten vivir o ayudar a vivir a las familias que tienen estas bodegas, sin más ambición que la "subsistencia".

- La llegada del primer centro comercial, situado en pleno centro tradicional, sería algo muy extraño en Europa, donde se protegen los centros históricos de las ciudades y su comercio. Sin embargo, aquí se considera como una novedad, y así lo es para ellos, ya que es el único centro comercial de la región. Ha sido instalado en el centro de la ciudad como símbolo de modernidad sin preocuparse del gran perjuicio que significa para el comercio tradicional y el fomento de la centralización con sus consecuencias como el atasco circulatorio y la dificultad que ocasiona para que los comerciantes $u$ otras actividades se animen a abrir en otras zonas de la ciudad.

Respecto a la comunicación debemos tener en cuenta:

- El tamaño de la ciudad, de unos 350000 habitantes, hace que funcionen bastante bien la recomendación y las ventas personales, por lo que se tiende a utilizar esta forma de comunicación para la comercialización, como ocurre en cualquier ciudad de este tamaño.

- La cultura y el idioma quechua, que es propio de los Andes, tienen la característica de ser oral, es decir no hay textos escritos. El castellano es el idioma dominante en Huancayo, el quechua es considerado un idioma en vías de extinción en esta ciudad, aunque no en el resto de los Andes; sin embargo, la cultura oral andina se mantiene. Por ello, el índice de lectura es muy bajo y hay pocos medios de comunicación escritos que sirvan de soporte de la publicidad.

- La combinación de los dos factores anteriores hacen que a las redes sociales les vaya muy bien como sustituto de la comunicación oral. A pesar de ello, se sigue sin hacer publicidad efectiva por los medios digitales. Como soporte publicitario, solo es utilizado el Facebook y no la página web ni otros medios.

\section{REFERENCIAS BIBLIOGRÁFICAS}

1. Puromarketing. 10 consejos de marketing local para un PYME [Internet]. [cited 2013 Oct 10]. Available from: www.puromarketing.com

2. AlarcónJ, etal. Comercializaciónagrícola en el Perú. Lima: Grupo de Análisis para el Desarrollo (GRADE) y Agencia para el Desarrollo Internacional (AID); 1994

3. Vignali C. McDonald's: "think global, act local" - the marketing mix. British Food Journal. 2001; 103(02):97-111. 